\title{
Klinische Praxis Aromataseinhibitoren: Upfront, Switch oder Extended?
}

\section{Clinical Practice Aromatase Inhibitors: Upfront, Switch or Extended?}

\author{
Gesprächsleitung: Nadia Harbeck (München) und Raimund Jakesz (Wien) \\ Teilnehmer: $\quad$ R. Greil, Salzburg W. Jonat / N. Maass, Kiel M. Kaufmann, Frankfurt/M. \\ O. Ortmann, Regensburg K. Possinger, Berlin G. Steger, Wien
}

Frage 1: Welchen Stellwert haben für Sie

Aromatasehemmstoffe in der adjuvanten Therapie beim Mammakarzinom?

Greil: Der Einsatz von Aromatasehemmstoffen in der adjuvanten Therapie des Mammakarzinoms hat in praktisch allen Studien bei postmenopausalen Hormonrezeptor (HR)-positiven Frauen aller Risikogruppen zu einer 20-40-prozentigen Reduktion des Rückfallrisikos geführt. Wichtig ist dabei, dass in der Mehrzahl der Studien, unter anderem in der ABCSG 8/ARNO Studie [Jakesz, Lancet 2005;366:455-462] auch das Fernmetastasierungsrisiko in ähnlichem Ausmaß reduziert wurde. Dies lässt zumindest für bestimmte Frauen auf eine lebensverlängernde Wirkung durch diese Therapie hoffen, auch wenn ein lebensverlängernder Effekt bis dato lediglich für die Subgruppe nodal positiver Frauen in der MA-17-Studie gezeigt werden konnte [Goss, New Engl J Med 2003;349: 1793-1802; Goss, Proc Am Soc Cin Oncol 2004;88s:847]. Die Datenlage für die Risikoreduktion postmenopausaler Frauen ist so eindeutig, dass das ASCO Technology Assessment Pannel geschlossen hat, dass die optimale adjuvante Therapie des HR-positiven Mammakarzinoms den Einsatz von Aromatasaehemmern als initiale Therapie oder im Anschluss an Tamoxifen einschließt [Winer, J Clin Oncol 2005;23:619-629]. Diese Aussage kann aus meiner Sicht derartig ergänzt werden, dass für jede postmenopausale HR-positive Frau als neuer Standard der Einsatz von Aromatasehemmstoffen exakt auf Sinn des Einsatzes geprüft werden muss und die standardmäßige Verschreibung von Tamoxifen nicht mehr als gerechtfertigt angesehen werden kann. Die Entscheidungsfindung ist jedoch komplex, da eine Fülle für den praktischen Einsatz relevanter Fragen wissenschaftlich nicht beantwortet ist:

\begin{tabular}{ll}
\hline KARGER & @ 2006 S. Karger GmbH, Freiburg \\
$\begin{array}{l}\text { Fax +49 761 45207 14 } \\
\begin{array}{l}\text { E-mail Information@Karger.de } \\
\text { www.karger.com }\end{array}\end{array}$ & $\begin{array}{l}\text { Accessible online at: } \\
\text { www.karger.com/brc }\end{array}$
\end{tabular}

- Es ist unbekannt, ob Aromatasehemmer die Elimination residueller HR-positiver Mikrometastasen erhöhen, zur Vermeidung von früher Hormonunabhängigkeit beitragen oder das Aufwachsen dormienter neoplastischer Zellen lediglich hinausschieben. Es bleibt also auf Grund der kurzen Nachbeobachtungszeit in den vorliegenden Studien und der Chronizität des Mammakarzinoms unklar, ob die Heilungsrate erhöht oder «lediglich» die Zeit bis zum Rezidiv verlängert wird.

- Es ist unklar, ob die Effekte über eine direkte Hemmung der Überlebensvorteile der Tumorzellen oder über eine Beeinflussung des Microenvironments der Tumorzellen ausgelöst werden, was für zukünftige Entwicklungen der adjuvanten Therapiekonzepte wichtig ist.

- Die Subgruppen mit höchster Benefit/Nebenwirkungsratio, die Methoden und die Prädizierbarkeit individuellen Benefits durch Mikroarrays, die exakten osteologischen, neurokognitiven und klinisch kardiovaskulären Nebenwirkungen sind vage bestimmt und angesichts der Überraschungen aus prospektiv randomisierten Studien zum Einsatz der Hormonersatztherapie im postmenopausalen Setting nicht sicher vorhersehbar [Espeland, JAMA 2004;291:2959-2968; Shumaker, JAMA 2004;291:2947-2958; Bath, BMJ 2005; 330:342; Koh, Arterioscler Thromb Vascul Biol 2004;7: 1171-1179].

Jonat/Maass: Die Aromatasehemmstoffe sollten Bestandteil der adjuvanten Therapie (Upfront, Switch oder «erweitert») beim hormonsensiblen, postmenopausalen Mammakarzinom sein.

Kaufmann: Die Einführung von Aromatasehemmstoffen ist ein wesentlicher Fortschritt in der Therapie postmenopausaler 
Frauen mit HR-positiven Tumoren. Die hierzu durchgeführten prospektiven Studien mit allerdings noch kurzer Beobachtungsdauer zeigen anhand großer Patientenkollektive mehrfach gleichartige Ergebnisse. Nahezu alle Frauen mit dieser Charakterisierung sollten im Laufe ihrer Behandlung einen Aromatasehemmstoff erhalten.

Ortmann: Bei der überwiegenden Anzahl der postmenopausalen Patientinnen mit HR-positivem Mammakarzinom sollten Aromatasehemmer eingesetzt werden. Aus meiner Sicht verdrängen sie Tamoxifen zunehmend, aber nicht vollständig aus der adjuvanten endokrinen Therapie.

Possinger: Aromatasehemmstoffe sind heute ein wesentlicher Bestandteil der adjuvanten antihormonellen Therapie von postmenopausalen Patientinnen mit hormonsensitiven Tumoren.

Steger: Aromataseinhibitoren und Aromataseblocker haben einen entsprechend großen Stellenwert, da sie bei postmenopausalen Patientinnen mit hormonabhängigem Mammakarzinom mittlerweile als Standardtherapie anzusehen sind.

Frage 2: Sollten alle HR-positiven postmenopausalen Patientinnen unmittelbar nach Operation $+/-$ Chemotherapie (d.h. "upfront") auf einen Aromatasehemmstoff eingestellt werden?

Greil: Wie erwähnt, sollte für alle Frauen geprüft werden, ob sie von der Anwendung eines Aromatasehemmstoffes profitieren können. Ich würde den sofortigen Upfront-Einsatz derzeit in jedem Fall für die Höchstrisikopatientinnen empfehlen. Diese Subgruppe hat auf Grund des hohen absoluten Risikos bei identischer relativer Risikoreduktion nicht nur den höchsten absoluten potentiellen Benefit. Aus den niedrigeren Risikoreduktionsraten der ATAC-Studie [ATAC Trialist's Group, Lancet 2002;359;2131-2139] (ca. 20\%) gegenüber Studien mit sequentiellem Switch-Design (ca. 40\% in der ABCSG-8/ ARNO Studie) kann auch geschlossen werden, dass bei einem Wechsel nach 2 oder 3 Jahren jene frühresistenten Patientinnen verloren gehen, die von einem frühen Einsatz der Aromatasehemmer profitieren könnten. Diese Ansicht wird indirekt gestützt durch die Überlegenheit der Aromatasehemmer gegenüber Tamoxifen im metastasierten Stadium [Mouridson, Oncologist 2004;9:489-496], aber auch durch die Subgruppenanalysen der MA-17-Studie zum späten Einsatz nach 5 Jahren Tamoxifen. Diese bescheinigen unter allen bisher durchgeführten Studien als einziger Gruppe den nodal positiven Frauen einen Überlebensvorteil, da die Höhe des Absolutrisikos im Beobachtungszeitraum Wirkunterschiede transparent machen kann [Goss, ASCO 2004, http://www.asco.org; Kaufmann, J Cancer Res Clin Oncol 2005;131:487-494]. Da dennoch nicht ausgeschlossen werden kann, dass der Einsatz von Aromatasehemmern besonders den Patientinnen mit dauerhaft hormonabhängigem, also biologisch besonders günstigem, Tumor und zusätzlichem klinischen Risiko wie Lymphknotenbefall (jährliches Rückfallrisiko 4 vs. 2\% für nodal negative Patientinnen [Pritchard, J Clin Oncol 2005;23: 4850-4852]) zu Gute kommt bzw. initiale Tamoxifen-Therapie zu einem «Priming» der Sensitivität gegenüber Aromatasehemmern führt, bleiben für sichere Aussagen die Studienresultate aus direktem Vergleich von «upfront» vs. «early switch» abzuwarten.

Auch wenn sich das ASCO Technology Assessment Panel nicht einheitlich für einen Einsatz bei bestimmten Subgruppen entscheiden konnte, sprechen die retrospektiven ATACDaten meines Erachtens deutlich für eine Bevorzugung einer Upfront-Aromatasehemmertherapie für Patientinnen mit $\mathrm{ER}^{+}, \mathrm{PR}^{-}$Tumor (annähernd doppelter Gewinn [Smith, Breast Cancer Treat Res 2003;82:\#1]). Da randomisierte Studien zur adjuvanten Therapie fehlen, spricht die Gesamtdatenlage unter Berücksichtigung neoadjuvanter Studien dafür, auch bei Her2/neu ${ }^{3+}$-Patientinnen den Upfront-Einsatz in Erwägung zu ziehen. Auch bei einer Kontraindikation für Tamoxifen stellen Aromatasehemmer das Mittel der Wahl für den Upfront-Einsatz dar.

Jonat/Maass: Da die Aromatasehemmstoffe in der UpfrontSituation einen Effektivitätsvorteil gegenüber Tamoxifen gezeigt haben, besteht prinzipiell eine Indikation bei jeder HRpositiven, postmenopausalen Patientin. Dennoch gibt es Patientinnen, bei denen ein primärer Einsatz mit Tamoxifen und ein späterer Switch, bzw. erweiterter Einsatz indiziert ist, mit vergleichbaren Effektivitätsergebnissen.

Kaufmann: Nahezu alle Frauen sollten eine Upfront-Aromatasehemmstoff-Therapie erhalten. Dies gilt insbesondere bei Tamoxifenunverträglichkeit bzw. Kontraindikation, bei HER2/neu-positiven Tumoren und bei einer Rezeptorkonstellation mit $\mathrm{ER}^{+}$und $\mathrm{PR}^{-}$Tumoren. Für Frauen mit sehr geringem Rezidiv- bzw. Todes-Risiko kann Tamoxifen nach wie vor upfront gegeben werden.

Ortmann: Derzeit halte ich es für nicht gerechtfertigt, alle HR-positiven, postmenopausalen Patientinnen unmittelbar nach Operation +/- Chemotherapie upfront mit einem Aromatasehemmer zu behandeln. Die Daten aus der BIGStudie sind positiv, aber noch zu unreif. Die Daten aus der ATAC-Studie weisen zumindest bei den Subgruppenanalysen, in die hohe Patientenzahlen eingeflossen sind, in einigen Situationen Resultate auf, die es wahrscheinlich machen, dass nicht jede Patientin von diesem Therapieregime profitiert.

Possinger: Derzeit ist noch unklar, ob die Upfront-Therapie bessere Ergebnisse erzielt als eine prognoseorientierte Behandlung. Klärung wird hierzu sicherlich die nächste Auswer- 
tung der BIG-Femta-Studie erbringen, wenn die sequentielle mit der Upfront-Gabe verglichen analysiert wird. Sicherlich muss auch dann bei den Patientinnen, die ein hohes Osteoporoserisiko oder schon einen Osteoporosenachweis haben, eine Tamoxifenbehandlung erwogen werden. Prinzipiell gehen wir derzeit prognoseorientiert vor. D.h. besteht ein hohes Rückfallrisiko (großer Tumor, viele befallene Lymphknoten, ungünstiges Grading usw.) oder besteht ein negativer PR-Status, so beginnen wir von vornherein mit einer Aromatasetherapie. Bei Patientinnen mit günstiger Prognose und insbesondere positivem PR wechseln wir 2-3 Jahre nach Tamoxifengabe auf einen Aromatasehemmer.

Steger: Nein, nicht automatisch alle Patientinnen. In Abhängigkeit vom individuellen Risikoprofil und evtl. Kontraindikationen sollen Aromatasehemmer entsprechend den vorliegenden Studiendaten entweder upfront, sequentiell nach 2 Jahren Tamoxifen oder nach 5 Jahren Tamoxifen eingesetzt werden.

\section{Frage 3: Welchen Patientinnen empfehlen Sie das "Fast/Switch»-Konzept (d.h. geplanter Wechsel auf einen Aromatasehemmstoff nach 2-3 Jahren Tamoxifen)? Gibt es Ihrer Meinung nach noch Patientinnen, die 5 Jahre mit Tamoxifen behandelt werden können?}

Greil: Ein Fast/Switch-Konzept empfehle ich für alle Patientinnen, bei denen nicht die Entscheidung zugunsten eines primären Einsatzes von Aromatasehemmstoffen fällt (siehe oben), und bei denen während der Therapie eine Unverträglichkeit gegen Tamoxifen aufgetreten ist. Es ist aber klar, dass die endgültige Entscheidung auf einer individuellen Basis erfolgen muss, bei der steigendes Rezidivrisiko und das Fehlen von Konstitutionen für substanzspezifische Komplikationen für den Einsatz von Aromatasehemmern sprechen, während ein eventuell besseres individuelles Nebenwirkungsprofil und ein extrem niedriges Rückfallrisiko eher für Tamoxifen sprechen. Für die alleinige Indikationsstellung zugunsten von Tamoxifen müssen daher nach derzeitiger Datenlage sehr gute Gründe vorliegen.

Jonat/Maass: Patientinnen, die sich z. Zt. unter Tamoxifentherapie befinden, sollten bis auf wenige Ausnahmen nach 2 Jahren auf einen Aromatasehemmer «geswitcht» werden. Primär geplante Switch-Patientinnen könnten insbesondere sowohl Frauen mit vorausgehender Chemotherapie oder ERund PR-positivem Status sein, als auch Patientinnen, die vom günstigen Nebenwirkungsprofil des Tamoxifens («Knochenschutz») profitieren. Die Patientinnen, die heute eine alleinige 5-jährige Tamoxifentherapie erhalten, sind sehr selten. Dies ist insbesondere für ältere Patientinen mit guter Prognose oder Patientinen, die nach den St. Gallen-Kriterien gar keine adjuvante Therapie benötigen und Tamoxifen mehr aus präventiven Gründen einsetzen, denkbar.
Kaufmann: Bis heute ist es unklar, ob das Switch-Konzept mit Tamoxifen oder einem Aromatasehemmer beginnen soll. Der Wechsel von zwei sehr unterschiedlich wirkenden Hormontherapien lässt günstige Ergebnisse erkennen. Theoretisch ist ein sogenanntes Tamoxifen-Priming sinnvoll. Eine 5-jährige Tamoxifen-Therapie hat auch heute noch Bedeutung, vor allem wenn Aromatasehemmer-induzierte Nebenwirkungen berücksichtigt werden müssen.

Ortmann: Dem überwiegenden Anteil der Patientinnen würde ich eine sequentielle Therapie mit einer 2- bis 3-jährigen Tamoxifengabe gefolgt von einer 2- bis 3-jährigen Gabe eines Aromatasehemmers empfehlen. Die besten Daten dazu stammen aus der IES-Studie. Der Grund für die Bevorzugung dieses Therapieregimes liegt zum einen darin, dass alle Subgruppen gleichermaßen von dieser Therapie profitieren. Zum anderen ist derzeit das Nebenwirkungsprofil dieser sequentiellen Therapie günstiger als das der Upfront-Behandlung. Aus meiner Sicht kommt noch ein kleiner Prozentsatz von Patientinnen für eine alleinige Tamoxifentherapie in Frage. Ein Beispiel dafür ist die ältere, postmenopausale Patientin mit einem niedrigen Risiko für ein Rezidiv.

Possinger: Alle Patientinnen mit günstiger Prognose, insbesondere solche Patientinnen mit positivem PR-Status und fehlenden Hinweisen für eine Osteoporose, werden nach 2-3 Jahren auf einen Aromatasehemmer umgestellt. Wahrscheinlich gibt es noch Patientinnen, die 5 Jahre mit Tamoxifen behandelt werden können. Definitive Antworten sind wiederum nach der BIG-Femta-Studie zu erwarten.

Steger: Allen Patientinnen ohne Kontraindikationen zu Aromatasehemmern soll der «Switch» angeboten werden. Bei Kontraindikation oder Unverträglichkeit gegenüber Aromatasehemmern ist Tamoxifen für 5 Jahre weiter eine valide Option.

\section{Frage 4: Empfehlen Sie allen Patientinnen, die 5 Jahre lang Tamoxifen eingenommen haben eine erweiterte adjuvante Therapie mit einem Aromatasehemmstoff?}

Greil: Dies ist wahrscheinlich die schwierigste Frage, da einerseits wie erwähnt das individuelle jährliche Rückfallrisiko für die Patientinnen über zumindest die ersten 15 Jahre konstant bleibt und damit eine 5-jährige Rezidivfreiheit zwar prognostisch günstig aber nicht prädiktiv für weitere Rezidivfreiheit ist. Die MA-17-Daten zeigen einerseits eine eindeutige Risikoreduktion, vor allem auch für Fernmetastasen, andererseits wird aber die Dauer der antihormonellen Therapie mit den daraus resultierenden Konsequenzen immer länger (ehedem 5 , demnächst $\geq 7,5$ Jahre). Da wir Frauen mit Benefit noch nicht ausreichend treffsicher identifizieren können und die absoluten Unterschiede zwischen den Subgruppen sehr klein 
sind, bestehen ernste Bedenken gegen die eventuell jahrzehntelange adjuvante Behandlung, die möglicherweise ohne tumorkausalen Nutzen ist, aber ein beträchtliches Nebenwirkungspotential hat. Folgende Faktoren sprechen meines Erachtens dennoch für einen Einsatz von Aromatasehemmern in dieser Situation:

- Evidenz für Spätresistenz durch das Fehlen eines Therapievorteils mit Tamoxifen über mehr als 5 Jahre und die klinische Evidenz für eine Reduktion der Spätresistenz durch Aromatasehemmer,

- hoher potentieller Benefit bei hohem Risiko für einen Rückfall, wie etwa nodale Positivität (siehe oben) usw., und

- ausreichende residuelle Lebenserwartung der Frauen auf Grund des Komorbiditätsprofils.

Wir versuchen derzeit sehr erfolgreich, möglichst alle Frauen für eine späte Rekrutierung in eine Aromatasehemmertherapie nach 5 Jahren Hormontherapie jeglicher Art im SALSAProtokoll der ABCSG zu gewinnen.

Jonat/Maass: Der erweiterte Einsatz von Tamoxifen nach 5 Jahren mit einem Aromatasehemmstoff sollte mit allen Patientinnen diskutiert werden. Letztendlich profitieren jedoch vorwiegend die bislang rezidivfreien Patientinen mit eher ungünstiger Prognose, insbesondere solche mit positivem Lymphknotenstatus.

Kaufmann: Grundsätzlich haben alle Patientinnen nach 5 Jahren Tamoxifen noch eine beachtenswerte Rückfallquote, wobei sich allerdings in retrospektiven Analysen die größeren Gewinne bei nodal positiven Frauen mit einem sogar signifikanten Überlebensvorteil aufzeigen ließen.

Ortmann: Die erweiterte adjuvante Therapie würde ich grundsätzlich jeder postmenopausalen Patientin anbieten, die eine 5-jährige Tamoxifentherapie abgeschlossen hat. Insbesondere kommen hier Frauen mit einem hohen Reszidivrisiko in Frage. Bei älteren postmenopausalen Patientinnen bin ich mit dieser Therapieempfehlung zurückhaltender.

Possinger: Alle Patientinnen werden von mir darüber informiert, dass es Hinweise dafür gibt, dass die Fortführung der antihormonellen Therapie mit einem Aromatasehemmer das rückfallfreie Überleben unabhängig vom Nodalstatus verlängert. Speziell weise ich bei Patientinnen mit primärem Lymphknotenbefall und positivem Rezeptorstatus nach 5 Jahren Tamoxifen darauf hin, dass unter diesen Bedingungen durch die Fortführung der Behandlung mit einem Aromatasehemmer sogar die Überlebenszeit signifikant verbessert wurde.

Steger: Ja, und optimalerweise im Rahmen der österreichischen ABCSG-16 (SALSA) Studie, in der die Dauer dieser erweiterten adjuvanten Therapie mit einem Aromatasehemmer geprüft wird.

\section{Frage 5: Wie differenzieren Sie zwischen den drei auf dem Markt befindlichen Substanzen? Welche Kriterien bestimmen Ihre Substanzwahl in der adjuvanten Therapie?}

Greil: Die relativen Risikoreduktionen für ein Lokalrezidiv, ein kontralaterales Rezidiv oder eine Fernmetastasierung sind für die verschiedenen Substanzen vergleichbar, sodass ein Klasseneffekt angenommen werden muss, auch wenn es individuelle Unterschiede in der Hemmung der Hormonproduktion sowie der androgenen Eigenwirkung gibt. Die bisherigen Studienresultate sprechen zwar dafür, dass diese biologischen Unterschiede klinisch unwirksam bleiben, dennoch kann man sich der Schlussfolgerung des ASCO Pannels anschließen, außerhalb von Studien in den jeweiligen Situationen jene Substanzen zu verwenden, die der jeweiligen in Studien ermittelten Datenlage am nächsten kommt. Daraus könnte folgender Schluss für den indikationsspezifischen Einsatz der Substanzen gezogen werden:

- Upfront-Therapie [ATAC, vs. Tamoxifen, BIG 01-98 vs.

Tamoxifen, Thürliman, http://www.ibcsg.org/public/documents/pdf/divers/BIG_1-98_stGallen_2005.pdf]: Anastrozol, Letrozol,

- Switch-Therapie [ABCSG/ARNO, ITA, IES]: Anastrozol, Exemestan

- später Wechsel [MA-17 Studie, vs. Placebo]: Letrozol

Es sollte allerdings auch die individuelle Erfahrung mit den verschiedenen Substanzen als Sicherheitsfaktor Eingang in die Entscheidung finden.

Jonat/Maass: $\mathrm{Zu}$ den unterschiedlichen Aromatasehemmern liegen bezüglich der Einsatzindikation unterschiedliche Studienergebnisse vor: Z.B. gibt es bislang keine Ergebnisse zur Switch-Therapie von Letrozol, bzw. keine Upfront-Ergebnisse zu Exemestan. Subgruppenanalysen, Publikationsstatus und Zulassungssituation spielen ebenfalls für den differenzierten Einsatz eine Rolle.

Kaufmann: Die Substanzwahl wird vorwiegend aufgrund einer Zulassung in Deutschland, durch das Nebenwirkungsprofil und den jeweiligen Einsatz in prospektiv-randomisierten Studien bestimmt.

Ortmann: Die drei Aromatasehemmer der 3. Generation, die in oraler Applikationsform zur Verfügung stehen, gehören grundsätzlich zu zwei verschiedenen Gruppen, den steroidalen bzw. den nicht-steroidalen. Die Substanzwahl wird im Wesentlichen durch die Qualität der Studienergebnisse und das in den Studien eingesetzte Therapieregime bestimmt.

Possinger: Für den therapeutischen Einsatz ist für mich die Zulassungsindikation und die Dauer der hierfür überblickbaren Studiendaten von grundlegender Bedeutung. Für die Upfront-Therapie kommen sowohl Anastrozol als auch Letrozol 
in Frage. Wegen der umfangreichen Datenmenge und des längeren Follow-up bevorzugen wir gegenwärtig Anastrozol.

Steger: Aus der Erfahrung in der Palliation sind sie derzeit als klinisch gleichwertig anzusehen. Somit setze ich die drei Substanzen entsprechend der vorliegenden Studiendaten ein.

\section{Frage 6: Halten Sie die auf dem letzten ASCO vorgestellten mathematischen Modelle für hilfreich bei der klinischen Indikationsstellung?}

Greil: Das beim ASCO vorgestellte Modell [Burstein, ASCO Educational 2005, p 40] behandelt naturgemäß die verschiedenen Studien statistisch gleichwertig und kann daher die biologischen Differenzen, die aus dem Design der Studien resultieren, nicht wettmachen. Es beruht zudem weitgehend auf 10Jahres-Vorhersagen von Studien mit jeweils deutlich kürzerer Nachbeobachtungszeit. Es unterstützt dennoch weitgehend die oben gemachten Aussagen, allenfalls mit der Ausnahme der Überbewertung der Fast/Switch-Verfahren. Es stellt eine brauchbare Hypothese für die wissenschaftliche Gemeinschaft dar, erlaubt aber keine individuelle Anpassung der Entscheidungsfindung im Alltag.

Jonat/Maass: Die mathematischen Modelle, obgleich sie zum Teil widersprüchliche Ergebnisse erbracht haben, sind interessante und hilfreiche Ergänzungen. Sie ersetzen jedoch keinesfalls die objektiven, prospektiv erhobenen Daten, die teilweise jedoch noch abgewartet werden müssen (insbesondere zum Gesamtüberleben).

Kaufmann: Die mathematischen Modelle sind äußerst interessant, aber für die klinische Indikationsstellung weniger hilfreich, da wir auch mit unseren theoretischen Überlegungen bzw. einer Kombination von Tamoxifen und Aromatasehemmer keinen Nutzen gegenüber einer alleinigen Tamoxifenbzw. Aromatasehemmertherapie gesehen haben.

Ortmann: Die mathematischen Modelle halte ich für wenig hilfreich. Für die klinische Indikationsstellung spielen die Resultate der Studien, sowie zum Teil auch das Verständnis der Tumorbiologie bzw. der individuellen Besonderheiten der Brustkrebserkrankung eine wichtigere Rolle.

Possinger: Die mathematischen Modelle sind in meinen Augen sehr hilfreich. Sie beleuchten die verschiedenen Sichtweisen des Einsatzes einer Aromatasehemmertherapie. Will man auf «Nummer sicher» gehen und macht sich keine Gedanken über die individuelle Prognose der Patientin, ist die Upfront-Therapie eine gute Modalität. Berücksichtigt man die prognostische Situation, was ich bevorzuge, so werden einem sowohl für die Upfront-Therapie als auch für den Behandlungswechsel nach 2-3 Jahren hilfreiche Daten an die Hand gegeben.
Steger: Derzeit sind sie für die Praxis nicht wirklich geeignet, doch ist auch der Bekanntheitsgrad dieser Modelle relativ gering.

\section{Frage 7: Stichwort Nebenwirkungen: Welche Neben- wirkungen der Aromatsehemmstoffe sind für Sie bei der adjuvanten Therapie klinisch relevant? Worüber sollten die Patientinnen aufgeklärt werden? Gibt es für Sie hier substanzspezifische Unterschiede?}

Greil: Die relevanten Nebenwirkungen, über die die Patientinnen auch aufgeklärt werden müssen, sind sicher der ausgeprägte Knochenverlust und die deutlich erhöhte Frakturrate gegenüber Tamoxifen. Dies muss im Abwägungsprozess der Nutzen-Risiko-Ratio bedacht werden. Zudem muss auf die Symptome des Östrogenentzuges in allen Konsequenzen hingewiesen werden.

Direkte Vergleiche der verschiedenen Aromatasehemmer, bzw. von steroidalen und nicht-steroidalen Aromatasehemmern stehen im Nebenwirkungsbereich ebenso noch aus wie im Hinblick auf die antitumorale Wirkung. Der indirekte Vergleich erlaubt aber derzeit keine Annahme eines klinisch relevanten Unterschiedes. Die Differenzen in den Thromboembolieraten gegenüber Tamoxifen sind in der ATAC-, der BIGund der IES-Studie jeweils signifikant und somit für alle 3 Substanzen valide; die relativen Risken sind vergleichbar. Ähnliches gilt für Arthralgie-Myalgie-Frequenz und die Vergleichbarkeit der kardiovaskulären Effekte. Grundsätzlich können Nebenwirkungsunterschiede aber nicht automatisch ausgeschlossen werden. Im indirekten Vergleich lassen sich Unterschiede in Hitzewallungen, Vaginalblutungen und Enodmetriumkarzinomen statistisch signifikant nur innerhalb der ATAC-Studie zugunsten des Aromatasehemmers beobachten. Diese Beobachtungen finden derzeit keine biologischen Erklärungen und sind bei ähnlichen Absolutwerten in der Inzidenz von fraglicher Relevanz.

Der Metabolit 17-Hydroxy-Exemestan hat eine androgene Eigenwirkung und kann damit dem Knochenverlust durch Östrogenreduktion eine Knochen aufbauende Eigenwirkung entgegensetzen, was im Tierversuch zu deutlichen Differenzen im Knochenaufbau im Vergleich zu etwa Letrozol führt. In klinischen Untersuchungen [Lonning, J Clin Oncol 2005;23: 5126-5137] bestätigt sich zwar laborchemisch eine Knochen aufbauende Aktivität, in der Summe überwiegt aber klinisch der Knochenverlusteffekt. Ein indirekter Vergleich der bisherigen Studien zeigt signifikant stärkere Reduktionen der Knochendichte mit Aromatasehemmern gegenüber Tamoxifen in allen Studien, mit Ausnahme der IES-Studie, die das nichtsteroidale Medikament Exemestan verwendetet. Eine Klärung des Sachverhaltes ist aber von der MA-27-Studie (http://www.ctsu.org) zu erwarten, die adjuvant Exemestan mit Anastrozol vergleicht und die Rate an Frakturen als sekundären Endpunkt einschließt. Inwieweit andere Effekte, wie 
beispielsweise neurokognitive Effekte aus der Langzeitanwendung und der unterschiedlichen Rolle androgener Eigenwirkung existieren, kann derzeit nur spekulativ behandelt werden.

Jonat/Maass: Das Nebenwirkungsprofil der Aromatasehemmer ist im Großen und Ganzen vergleichbar. Die Patientinnen müssen insbesondere über die nicht seltenen Muskelbeschwerden und Knochenproblematik aufgeklärt werden.

Kaufmann: Klinisch relevante Nebenwirkungen sind: Osteoporose, mögliche Kardiotoxizität, Lipidstoffwechselveränderungen und eine mögliche Beeinträchtigung kognitiver Fähigkeiten, wofür es allerdings noch keine Langzeitdaten gibt. Dies muss bei einer Aufklärung berücksichtigt werden. Substanzspezifische Unterschiede scheinen marginal, obwohl zwischen nicht-steroidalen und steroidalen Aromatasehemmern unterschieden werden muss.

Ortmann: Die klinisch relevanten Nebenwirkungen von Aromatasewirkstoffen resultieren zu einem großen Teil aus dem kompletten Östrogenentzug. Eine der wichtigsten Nebenwirkungen stellt hier für mich die Steigerung der Osteoporoserate sowie der daraus resultierenden Frakturen dar. Weitere östrogenmangelbedingte Nebenwirkungen sind die Urogenitalatrophie sowie Hitzewallungen und Schweißausbrüche. Zudem können Gelenkbeschwerden auftreten, die nach meiner Erfahrung im praktischen Umgang mit Aromatasehemmstoffen häufiger sind als unter einer Tamoxifentherapie. Dies entspricht auch den Studienergebnissen. Die Patientinnen sollten behutsam über all diese Nebenwirkungen aufgeklärt werden. Es gibt substanzspezifische Unterschiede, wesentlicher sind allerdings die Unterschiede, die auf Grund der verschiedenen Therapieregime entstehen (5 Jahre Tamoxifen vs. 5 Jahre Aromatasehemmer vs. 2-3 Jahre Tamoxifen gefolgt von 2-3 Jahren Aromatasehemmern).

Possinger: Prinzipiell mache ich mir bei Patientinnen mit osteoporotischen Knochenschmerzen große Gedanken, bevor ich Aromatasehemmer + Kalzium + Vitamin D + Bisphosphonate + kalziumreiche Kost empfehle. Des Weiteren berücksichtige ich dabei vorbestehende Beschwerden hinsichtlich Trockenheit der Haut und der Schleimhäute. Rein subjektiv würde ich auch bei Patientinnen mit kardial auffälliger Anamnese eher Anastrozol als Letrozol oder Exemestan einsetzen. Doch gibt es hierfür letztendlich keine ausreichende Datenlage, sondern nur ein gefühlsbedingtes Handeln. Substanzspezifische Unterschiede hinsichtlich der therapeutischen Wirksamkeit halte ich eher für marginal.

Steger: Es gibt keine substanzspezifischen Unterschiede. Im Vordergrund stehen sicher die muskuloskeletalen Symptome, die bei ca. $10-15 \%$ der Patientinnen auftreten und oft schwierig zu behandeln sind, sowie die Osteoporose mit der Notwendigkeit der Bisphosphonatprophylaxe bzw. -therapie. Man soll aber auch mögliche kognitive Störungen beachten, die allerdings noch wenig untersucht sind.

\section{Frage 8: Welche zusätzlichen Untersuchungen empfehlen Sie routinemäßig während einer Therapie mit Aromatasehemmstoffen?}

Greil: Es sollte eine initiale Knochendichtemessung erfolgen, zumindest die ASCO-Empfehlungen zur Prävention von Osteoporose und Frakturen eingehalten und bei kritischer Abnahme der Knochendichte der Einsatz von Bisphosphonaten in Erwägung gezogen werden. Risikofaktoren für Thromboembolien, kardiovaskuläre Komplikationen usw. sollten gemäß den allgemeinen internistischen Empfehlungen altersgemäß und risikoangepasst überwacht werden. Die übrigen Untersuchungen entsprechen weitgehend den ASCO Empfehlungen.

Jonat/Maass: Bei symptomatischen Patientinnen (z. B. Patientinnen mit Osteoporoserisiko) können jährliche Knochendichtemessungen empfohlen werden. Generalisierte, zusätzliche Untersuchungen im Zusammenhang mit der Aromatasehemmertherapie empfehlen wir jedoch nicht.

Kaufmann: Hinsichtlich eines Osteoporoserisikos sind vor allem anamnestische Untersuchungen wichtig. Im Einzelnen sind die ASCO-Empfehlungen risikoadaptiert zu übernehmen. Anamnestische Erhebungen sind bzgl. Kardiotoxizität und Thromboserisiko erforderlich.

Ortmann: Bei jeder Patientin sollte vor Beginn der Therapie mit einem Aromatasehemmer eine Knochendichtemessung durchgeführt werden. Bei kardiovaskulären Erkrankungen empfehle ich der Patientin eine Mitbetreuung durch einen Kardiologen und gegebenenfalls zusätzliche medikamentöse Maßnahmen.

Possinger: Vor Therapieeinleitung und unter Therapie führen wir Knochendichtebestimmungen durch. In der Regel genügen als therapeutische Maßnahme die Empfehlung von kalziumreicher Kost und Bewegung an der frischen Luft. Häufig sind nach 2-3 Jahren Therapie dann doch Verordnungen von Kalzium und Vitamin-D-Präparaten vorübergehend erforderlich. Nur relativ selten bedingen die ermittelten Messwerte eine zusätzliche Bisphosphonattherapie (T-Score).

Steger: Basis-Knochendichtemessung und jährliche Kontrollen. 
Frage 9: Würden Sie auch eine prämenopausale Patientin adjuvant mit einem Aromatasehemmstoff behandeln?

Greil: Aromatasehemmstoffe sind nicht geeignet um bei prämenopausalen Patientinnen mit intakter Ovarialfunktion die Östrogenproduktion ausreichend zu hemmen, sie können durch Stimulation der Gonadotropin-Freisetzung Ovulationen auslösen, sodass bei funktionell prämenopausalen Patientinnen berechtigte Bedenken einer kontraproduktiven Tumorzellstimulation bestehen und eine Kontraindikation ausgesprochen werden muss.

Schwieriger ist die Situation bei an sich prämenopausalen $\mathrm{Pa}-$ tientinnen mit chemotherapieinduzierter Amenorrhoe. Bestand die Amenorrhoe länger als 12 Monate, war sie die Folge der Chemotherapie und waren die FSH-Werte im postmenopausalen Bereich wurde eine sehr kleine Zahl von Patientinnen in die ATAC Studie eingeschlossen. Eine sichere Aussage zu dieser Konstellation kann auf Grund der geringen Datenlage nicht gemacht werden, das Problem ist quantitativ klein. Besteht jedoch ein sehr hohes Rückfallrisiko kann die Situation mit der Patientin besprochen werden. Diese Frauen würden sich jedenfalls für einen Fast/Switch-Ansatz eignen. Bleiben sie unter Tamoxifen amenorrhoisch, kann bzw. sollte aus meiner Sicht mit wohl ausreichender Sicherheit nach 2 Jahren auf einen Aromatasehemmer gewechselt werden.

Die Kombination eines Aromatasehemmstoffes mit einem GnHRH-Analogon ist nahe liegend und im metastasierten Setting nach eingetretener Resistenz auf die Kombination von GnHRH-Analogon mit Tamoxifen klinisch erfolgreich. Klinische und endokrine Daten sprechen auch bei prämenopausalen Patientinnen für eine Wirksamkeit dieses Ansatzes, der in der ABCSG 12-Studie prospektiv im Vergleich zu einer Kombination mit Tamoxifen getestet wird. Die IBSG 25-02 TEXTStudie hat ein identisches Design, ersetzt aber Goserelin durch Triptorlein und Anastrazol durch Exemestan. Auch in der IBCSG 24-02-SOFT-Studie wird bei chemonaiven oder postchemotherapeutisch prämenopausalen Patientinnen der Vergleich von Tamoxifen mit Ovarialsuppression plus Tamoxifen oder Exemestan gezogen. Die Ergebnisse dieser Studien bleiben abzuwarten, bevor im klinischen Alltag außerhalb von Studien prämenopausale Patientinnen so behandelt werden.

Jonat/Maass: Die Aromatasehemmer sollten bei prämenopausalen, HR-positiven Patientinnen in der adjuvanten Situation zum jetzigen Zeitpunkt nicht eingesetzt werden. Hier empfehlen wir die Teilnahme an den in Deutschland bereits laufenden Studien ABCSG-12, SOFT, TEXT, PERCHE.

Kaufmann: Prämenopausale Patientinnen dürfen derzeit nur in Studien mit Aromatasehemmstoffen behandelt werden.

Ortmann: In der klinischen Routine empfehle ich die Behandlung mit einem Aromatasehemmer von prämenopausalen $\mathrm{Pa}$ - tientinnen nicht. In Ausnahmefällen (z.B. bei Unverträglichkeit bzw. Kontraindikation gegen eine Tamoxifentherapie) wende ich Aromatasehemmer nach Ovarrektomie bzw. Therapie mit GnRH-Analoga an. Vorzugsweise werden wir diese Patientinnen jedoch in die von Herrn Prof. Kaufmann und mir in Deutschland koordinierten SOFT- / TEXT- und PERCHE-Studien einschließen.

Possinger: Wir wissen, dass der Östrogenentzug (Ausschaltung der Ovarialfunktion) therapeutisch sehr gut wirksam ist. Die konsequente Unterdrückung der übrigen Östrogenproduktion durch Aromatasehemmer wäre also nur logische Konsequenz. Allerdings fehlen hier die Daten klinischer Studien. Möglicherweise gilt auch hier, dass bei Patientinnen mit günstigerer Prognose die Kombination von Ovarsuppression und Tamoxifengabe zur Prävention rascher Osteoporoseentwicklung sinnvoller ist als die gleichzeitige Gabe von GnrH-Analoga und Aromatasehemmern.

Steger: Derzeit empfehle ich Aromatasehemmer prämenopausalen Patientinnen nicht, da die entsprechenden Studienergebnisse fehlen. ABCSG-12 wird diese Daten aber in naher Zukunft generieren.

\section{Teilnehmer}

Univ.-Prof. Dr. Richard Greil

III. Medizinische Universitätsklinik

Müllner Hauptstrasse 48, 5020 Salzburg, Österreich

Prof. Dr. Walter Jonat

PD Dr. med. Nicolai Maass

Klinik für Gynäkologie und Geburtshilfe

Universitätsklinikum Schleswig-Holstein Campus Kiel

Michaelisstr. 16, 24105 Kiel, Deutschland

Prof. Dr. med. Manfred Kaufmann

Klinik für Frauenheilkunde und Geburtshilfe

Johann Wolfgang Goethe-Universität

Theodor-Stern-Kai 7, 60590 Frankfurt/M., Deutschland

Prof. Dr. med. Olaf Ortmann

Klinik für Frauenheilkunde und Geburtshilfe

Universität Regensburg

Caritas-Krankenhaus St. Josef

Postfach 1006 45, 93006 Regensburg, Deutschland

Prof. Dr. med. Kurt Possinger

Medizinische Klinik und Poliklinik

Uniklinikum der Humboldtuniversität zu Berlin

Campus Charite Mitte

Schumannstraße 20/21, 10117 Berlin, Deutschland

Univ.-Prof. Dr. Günther Steger

Medizinische Universität Wien

Klinische Abteilung für Onkologie

Währinger Gürtel 18-20, 1090 Wien, Österreich 